\title{
Las adaptaciones curriculares
}

Autor: Reta Sabarrós, Amaia (Maestro Especialidad en Educación Especial, Licenciada en Psicopedagogía, Graduada en Educación Primaria, Maestra de Pedagogía Terapéutica).

Público: Maestros tutores, maestros de apoyo, Pedagogía Terapéutica. Materia: Atención a la diversidad. Idioma: Español.

Título: Las adaptaciones curriculares.

Resumen

Con los planteamientos curriculares recogidos en la Ley Orgánica 8/2013, de 9 de diciembre, para la Mejora de la Calidad Educativa, LOMCE, aparece un modelo de escuela abierto a la diversidad, donde se pretende que todas las personas obtengan respuestas a sus peculiaridades y a sus necesidades especiales. Abordaremos a continuación una de las estrategias más importantes que, desde los diferentes niveles de concreción curricular, el profesorado puede llevar a cabo para dar una respuesta a la diversidad: las adaptaciones curriculares.

Palabras clave: Adaptación curricular, atención a la diversidad, necesidades educativas especiales.

Title: Curricular adaptations.

Abstract

With the curricular approaches gathered in the Organic Law 8/2013, of December 9, for the Improvement of Educational Quality, LOMCE, a model of a school open to diversity appears, where it is intended that all people obtain answers to their peculiarities and their special needs. We will then approach one of the most important strategies that, from the different levels of curriculum concretion, teachers can carry out to give a response to diversity: curricular adaptations.

Keywords: Curricular adaptation, attention to diversity, special educational needs.

Recibido 2016-11-30; Aceptado 2016-12-12; Publicado 2016-12-25; Código PD: 078104

\section{INTRODUCCIÓN}

Cuando hablamos de adaptar la enseñanza o el currículo «no nos referimos sólo al diseño de un programa que dé respuestas a niños con "necesidades especiales", sino que nos referimos a un diseño del currículo en el cual tengan cabida todos los niños, tanto los que no presentan ningún problema como los que presentan algún problema en un momento determinado en su proceso de aprendizaje, así como los que presentan déficits, o los que son superdotados» (Córdoba, 1991:60).

Adaptar el currículo supone hacerlo asequible a todos los alumnos, y hablar de un currículo común no supone una contradicción con la atención a la diversidad. A pesar de hablar de un currículo único para todos, éste debe dejar margen a la adaptación y flexibilización de sus elementos, para dar una respuesta educativa adecuada y eficaz a todos. Esta respuesta se debe ir concretando en determinadas medidas de atención a la diversidad en los diferentes niveles curriculares.

\section{PLANIFICACIÓN DEL PROCESO DE ATENCIÓN A LA DIVERSIDAD A PARTIR DE LA ADAPTACIÓN DE LOS DIFERENTES NIVELES DE CONCRECIÓN CURRICULAR}

El primer nivel de concreción curricular lo constituye el Diseño Curricular Base. Este documento, elaborado por el Ministerio de Educación, Cultura y Deporte, recoge la propuesta curricular que, a nivel nacional, se plantea. En él se recogen las intenciones educativas y los elementos comunes que debe desarrollar la enseñanza en todos los alumnos. A pesar de la necesidad de un currículo común, que dé continuidad y coherencia a las diferentes etapas del sistema educativo, éste debe ser abierto y flexible, para garantizar una respuesta educativa en función de las diferencias individuales. "Esta propuesta debe ser, sin embargo, suficientemente abierta como para permitir la intervención de las Comunidades Autónomas con competencias educativas y su adecuación a la realidad del contexto socioeconómico y cultural de cada centro escolar y a las características específicas de sus alumnos. Su carácter abierto y flexible permitirá respetar el pluralismo cultural y dar una respuesta educativa que tenga en cuenta la diversidad de capacidades, intereses y motivaciones de los alumnos» (MEC, 1989). 
Podemos decir que ésta sería la primera adaptación que se realiza del currículo básico, es decir, la que llevan a cabo cada una de las Comunidades Autónomas con competencias en materia educativa, con el fin de adecuar o adaptar las prescripciones generales a sus distintas realidades.

El segundo nivel de concreción curricular lo constituye el Proyecto Educativo de Centro. Esta planificación corresponde al centro en su conjunto y debe favorecer la participación de todos los miembros implicados. Cuando se planifica la acción educativa se debe tener en cuenta el contexto que rodea al centro, la cultura propia de éste, las características y necesidades de los alumnos, el profesorado y los servicios de apoyo.

Las medidas de atención a la diversidad que va a desarrollar el centro deben quedar recogidas en dicho documento, ya que dentro del marco de la LOE (2006) se contempla que la atención a la diversidad se aborde, no de forma independiente, sino dentro del Proyecto Educativo de Centro y de las Concreciones Curriculares, de tal forma que cada centro adapte de manera adecuada las orientaciones generales a su realidad escolar, potenciando así la autonomía e identidad de cada centro. Por eso, cada escuela debe tener un Proyecto Educativo diferente al de las demás escuelas, ya que deben responder a necesidades distintas. El Proyecto Educativo debe convertirse en un instrumento dinámico y útil para toda la comunidad escolar, en lugar de ser un documento enfocado al puro trámite burocrático de los centros.

Según Gairín (1998), un Proyecto Educativo preocupado por atender a la diversidad del alumnado debe tener en cuenta los siguientes planteamientos:

- Sensibilizar a toda la comunidad escolar de la necesidad de educar en la diversidad, implicando a todos los miembros de la organización en el desarrollo del proceso.

- Favorecer el contacto con otros centros e instituciones, que aporten realidades diferentes.

- Desarrollar una relación estrecha entre el centro, el entorno y las familias.

- Educar en el respeto a las diferencias, la solidaridad y la tolerancia. Un Proyecto Educativo que apueste por la diversidad debe contemplar y desarrollar los valores y actitudes acordes con ésta.

- Incorporar en el currículo contenidos y objetivos relacionados con la diversidad cultural, personal y social de los alumnos.

- Fomentar la formación permanente del profesorado para atender a la diversidad. Los docentes deben estar preparados para poder dar respuesta a las necesidades e intereses de todos sus alumnos; por ello el centro debe impulsar su continua formación.

Dentro del Proyecto Educativo, además de otros documentos, encontramos las Concreciones Curriculares. Constituyen la respuesta curricular que el centro ofrece a los alumnos, concretada en cada una de las etapas. Si dichas Concreciones pretenden atender a la diversidad, debe responder a las siguientes cuestiones (Jové, 2001):

- ¿ ¿Qué enseñar? Los objetivos y contenidos del currículo deben acomodarse a las características de cada centro y de sus alumnos. Para ello, se deben tomar medidas para flexibilizar dichos elementos.

- ¿Cuándo enseñar? Se debe prever una organización flexible del espacio y el tiempo, que atienda tanto a las preferencias personales en relación con la forma de trabajar, como a diferencias en el ritmo de aprendizaje y a posibles dificultades.

- ¿Cómo enseñar? Se ha comprobado que existen algunos métodos más adecuados para los alumnos con N.E.E. (Sáenz, 1994; Pújolas et al., 1997; Federico, Herrold y Venn, 1999; Masip y Rigol, 2000; Sanahuja, 2000) como son: la enseñanza individualizada, el trabajo cooperativo, la tutoría entre iguales, el trabajo por proyectos y por talleres, etc.

Adecuar la metodología implica también flexibilizar las actividades y experiencias de aprendizaje, los medios, los recursos, los apoyos, los espacios, los tiempos, los aspectos organizativos, el agrupamiento de los alumnos, etc. Lo que ha de quedar claro es que no existe una metodología «ideal», sino que todo depende de las características y capacidades del alumno. Se aconseja partir de lo que los alumnos/as conocen y proponerles tareas al alcance de sus posibilidades.

- ¿QQué, cómo y cuándo evaluar? En el momento de la evaluación es necesario tener en cuenta: el alumno y el proceso de enseñanza-aprendizaje. 
Todo esto debe desembocar y reflejarse en el último nivel de concreción curricular, la programación de aula. La programación se define como «conjunto de unidades didácticas ordenadas y secuenciadas para las áreas de cada ciclo educativo. Esta tarea exige dos pasos fundamentales: planificar y distribuir los contenidos de aprendizaje a lo largo de cada ciclo; y planificar y temporalizar las actividades de aprendizaje y evaluación correspondientes» (MEC, 1989). Según esto, se podría afirmar, al igual que Arnáiz y Garrido (1997), que dentro de cada aula existen dos niveles de elaboración curricular. Uno, referido a todos los alumnos de un mismo ciclo, concretado en cada una de las aulas, que recogería los objetivos generales para el ciclo, la secuenciación coherente de contenidos, la metodología y actividades a desarrollar y los criterios de evaluación. Otro, que sería propiamente la programación de aula, es decir, la secuenciación ordenada de unidades didácticas, instrumento que posibilita al profesorado articular el proceso de enseñanza-aprendizaje de forma eficaz. En ambos niveles deben estar contempladas las diferentes adaptaciones que se van a desarrollar para dar respuesta a las necesidades de los alumnos. Dichas adaptaciones tendrán su referente en el Proyecto Educativo y en las Concreciones Curriculares, como signo de coherencia y continuidad.

Este nivel de concreción curricular es fundamental en el proceso de atención a la diversidad, es decir, el aula ordinaria, ya que es en ella donde van a tener lugar las experiencias de enseñanza-aprendizaje. Un aula inclusiva es aquella en la que todos los niños pertenecen al grupo y pueden aprender en el aula ordinaria. Cada miembro tiene unos derechos; el principal, ser tratado de forma justa e igual, con respeto. Por eso, cada alumno conseguirá unos objetivos curriculares adecuados a sus capacidades, para lo cual el currículo se adapta, se amplía o ambas cosas.

A continuación se presenta un esquema en el que se recogen los diferentes niveles de concreción curricular.

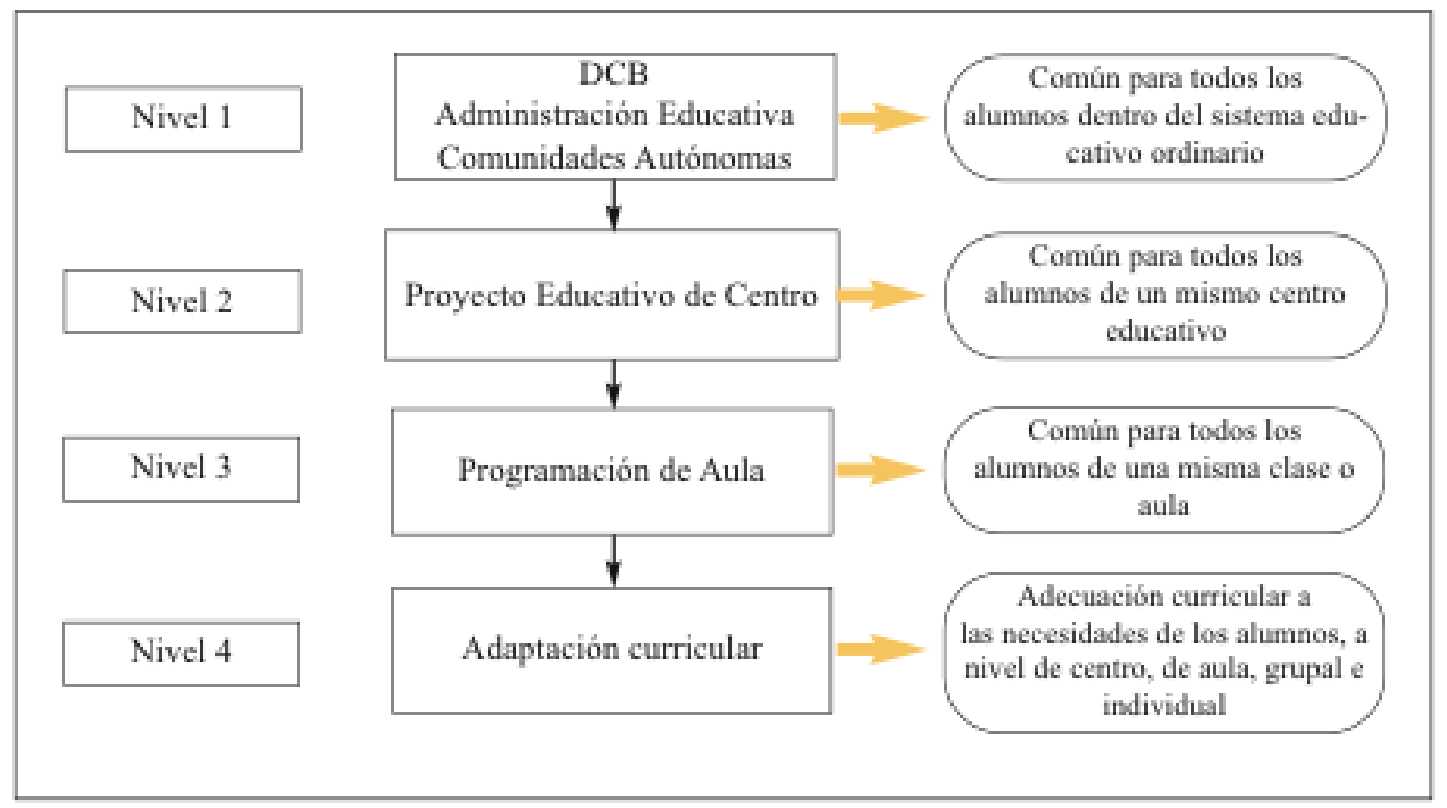

\section{RESPUESTAS A NECESIDADES EDUCATIVAS ESPECIALES}

Desde el currículo podemos encontrar diversas estrategias para responder a las necesidades educativas especiales; una de ellas pueden ser las adaptaciones curriculares.

La adaptación curricular, según Porras (1998), se establece como un continuo en el proceso educativo para dar respuesta a la diversidad, dentro del marco abierto y flexible que propone la LOMCE. Pero esta adaptación encuentra una serie de obstáculos que es conveniente tener en cuenta (García Pastor, 2000):

- El modelo de enseñanza ha de cambiar, pero para ello es necesario argumentar de manera seria y rigurosa las ventajas que ofrece un modelo comprensivo de escuela, tanto a profesores como a alumnos.

- No está muy claro lo que significa el diseño curricular base como orientación prescrita y la flexibilización de éste para que sea accesible a todos. 
- Aparecen contradicciones entre la idea de un currículo común y la idea de que no es posible enseñar lo mismo a niños diferentes.

- Es incongruente pretender desarrollar un currículo flexible, adaptado a las necesidades de los alumnos, dentro de instituciones con estructuras organizativas inflexibles y rígidas.

\subsection{Concepto y principios}

De las vías y estrategias de atención a la diversidad que los profesionales aplicarán en los centros de educación primaria, las adaptaciones curriculares son una de las más utilizadas.

Las adaptaciones curriculares se definen como "el conjunto de modificaciones que se realizan en los objetivos, contenidos, criterios y procedimientos de evaluación, actividades y metodología para atender a las diferencias individuales de los alumnos» (MEC, 1996).

Las adaptaciones curriculares son estrategias de adecuación del currículo general a las necesidades, peculiaridades y características individuales de los alumnos. Estas adaptaciones van a permitirnos dar un paso más en la individualización del proceso de enseñanza-aprendizaje de aquellos alumnos que presenten necesidades educativas especiales desde la programación de aula. A través de ellas podremos ajustar y acomodar la oferta educativa a las posibilidades y necesidades de cada alumno.

La adaptación curricular es un proceso de toma de decisiones sobre los elementos curriculares cuya finalidad es que todos los alumnos puedan acceder al currículo. Las adaptaciones curriculares se llevarán a cabo atendiendo y teniendo en cuenta:

- Las necesidades de los alumnos.

- Las circunstancias y posibilidades del centro.

- Los medios y recursos disponibles (López Melero, 1990).

Según la Dirección General de Ordenación Educativa y Formación Profesional (1994), estas adaptaciones deben atender a los siguientes principios:

- Normalización. No se debe perder de vista que toda adaptación curricular tiene como referente el currículo ordinario, ya que lo que se pretende es desarrollar un proceso educativo normalizado.

- Contextualización. Es necesario que la adaptación curricular se adecue a las necesidades de los alumnos, y para ello es imprescindible que se tenga un conocimiento lo más objetivo posible de las características del entorno, del centro educativo, del grupo de alumnos y más concretamente de cada alumno en particular. Para ello, se debe realizar una evaluación inicial para poder conocer cuál es la situación real.

- Significatividad. La adaptación curricular debe partir de lo menos significativo, es decir, debe comenzar por adaptar los elementos de acceso al currículo, para pasar más tarde, si es necesario, a adaptar aquellos elementos considerados como básicos dentro de éste.

- Realidad. Está claro que la formulación de la adaptación curricular debe ser realista, y a que de lo contrario se caería en un falso idealismo. Se debe tener presente: los recursos existentes, los elementos que se deben adaptar, los objetivos que se pueden conseguir, etc.

- Participación e implicación. Para llevar a cabo una adaptación curricular es necesario que se impliquen en dicho proceso el tutor, el profesor de apoyo, el equipo docente y todos aquellos servicios, tanto internos como externos al centro, que sean necesarios. Ésta no es una labor individual, sino de equipo.

Las diferencias individuales no se presentan de forma homogénea, sino que se manifiestan con grados diferentes, por lo que las adaptaciones curriculares deben ser graduales y situarse en un continuo que abarque todas las situaciones (Salvador, 1998). 


\subsection{Niveles de adaptación curricular}

Con el planteamiento curricular propuesto por la LOE (2006) podemos analizar tres niveles de adaptación curricular. Siguiendo la Orden por la que se regula el procedimiento de diseño, desarrollo y aplicación de adaptaciones curriculares (1994), los niveles son:

1. Para el centro: van destinadas a todos los alumnos del centro.

2. Para el aula: Programación de aula. Van destinadas a todos los alumnos de un grupo-clase, teniendo en cuenta las N.E.E. de algunos alumnos.

3. Para el alumno concreto: Adaptación curricular individualizada; dirigida a aquellos alumnos con necesidades educativas especiales.

Al detenernos en los niveles de adaptación curricular observamos que están en estrecha relación con los niveles de concreción curricular descritos anteriormente, que a su vez vienen a coincidir con los tipos de adaptaciones curriculares.

\subsection{Adaptaciones curriculares individualizadas}

Al clasificar las adaptaciones en función del grado de significación nos encontramos con un continuo que oscila entre lo poco significativo a lo muy significativo. En el primer grupo estarían aquellas modificaciones en los elementos de acceso que van a permitir al alumno desarrollar las capacidades enunciadas en los objetivos generales de etapa sin prácticamente variar la Programación de Aula: metodología, actividades y recursos. Serán consideradas como más significativas las adaptaciones que afecten a los elementos prescriptivos curriculares: objetivos, contenidos básicos y criterios de evaluación.

Las adaptaciones en los elementos de acceso, por ser menos significativas no son menos importantes, ya que en ocasiones el hecho de adaptar elementos de acceso convenientemente puede hacer innecesario cualquier otro tipo de adaptación.

A continuación vamos a explicar con mayor detenimiento cada uno de los elementos en las adaptaciones curriculares individualizadas.

a) Adaptaciones en los elementos de acceso

Adecuación de los elementos humanos

\begin{tabular}{|l|l|}
\hline \multicolumn{1}{|c|}{ Elementos personales } & \multicolumn{1}{|c|}{ Elementos de adecuación } \\
\hline Grupo de alumnos & $\begin{array}{l}\text { - Flexibilidad del agrupamiento según actividades. } \\
- \text { Mantener la heterogeneidad de los grupos. } \\
- \text { Organizar estrategias de trabajo cooperativo. }\end{array}$ \\
\hline Tutores & $\begin{array}{l}\text { - Trabajo en equipo con los tutores del ciclo y el profe- } \\
\text { sorado especialista y de apoyo. }\end{array}$ \\
\hline Equipo técnico de coordinación pedagógica & $\begin{array}{l}\text { - Asegurar y posibilitar la flexibilidad de horarios y el } \\
\text { aprovechamiento de los recursos humanos existentes. }\end{array}$ \\
\hline Equipo de orientación y apoyo & $\begin{array}{l}- \text { Organización y distribución de funciones en base de } \\
\text { la adaptación curricular realizada. }\end{array}$ \\
\hline $\begin{array}{l}\text { Equipo de Orientación Educativa y Psicope- } \\
\text { dagógica }\end{array}$ & - Pactar el epplan de acción» con el ETCP. \\
\hline Otros profesionales & $\begin{array}{l}\text { - Coordinación con el profesor tutor. } \\
\text { - Asunción de sus competencias. }\end{array}$ \\
\hline
\end{tabular}




\section{Adaptaciones en los espacios y aspectos físicos}

Son las que se refieren a la facilitación del uso de las instalaciones del centro y el desenvolvimiento de los alumnos en las mismas. Se refieren a:

- Accesos al centro y movimiento por el mismo: eliminación de barreras arquitectónicas.

- Ubicación del alumno en el aula.

- Disposición del mobiliario y regularidad en su colocación.

- Adecuación de los espacios, tanto para el trabajo en grupo como para la atención individual.

- Condiciones físicas de los espacios.

- Instalar indicadores y señales por el centro para facilitar la orientación de los alumnos con necesidades educativas especiales.

- Designar espacios concretos para apoyos específicos fuera del aula.

\section{Adaptaciones en el equipamiento y los recursos}

Se refiere a la variedad y riqueza de materiales didácticos que hacen posible la mayor diversidad de experiencias. Adaptar los materiales supone:

- Disponer del equipamiento y recursos didácticos suficientes y adecuados a las necesidades de los alumnos.

- Crear y confeccionar materiales que no se encuentren en el mercado.

- Crear un aula de recursos en la que confluyen los diferentes departamentos didácticos del centro.

- Dotarse de materiales y recursos que sean igualmente válidos para alumnos con necesidades educativas especiales que para el resto.

\section{Adaptación del tiempo}

- Decisión del tiempo de cada área y su equilibrio.

- Adecuación del tiempo dentro y fuera del aula.

- Tiempo fuera del horario lectivo.

b) Adaptaciones de los elementos básicos del currículo

\section{Adaptaciones en los objetivos}

Es posible adaptar los objetivos de área, siempre y cuando esto no suponga una modificación en los objetivos generales de etapa. Es posible, atendiendo a las necesidades especiales del alumno, una reformulación de los objetivos, una secuenciación y priorización de los mismos.

Arnáiz y Garrido (1997:62) plantean una serie de sugerencias con respecto a la adaptación en los objetivos:

- Tener en cuenta a los alumnos del centro con necesidades educativas especiales a la hora de adecuar los objetivos generales de etapa y concretar los de área en los distintos ciclos.

- Introducir objetivos y contenidos que, aunque formando parte del contenido curricular del centro, no se encuentren contemplados dentro de la etapa en la que se hallan los alumnos. 


\section{Adaptaciones metodológicas}

Son las modificaciones que se realizan en los agrupamientos de alumnos; los métodos, técnicas y estrategias de enseñanza-aprendizaje y evaluación y las actividades programadas.

Algunos ejemplos:

- Establecer criterios metodológicos comunes para los ciclos o niveles educativos que favorezcan una respuesta lo más normalizada posible a las N.E.E.

- Situar a los alumnos con N.E.E. en los grupos donde mejor puedan trabajar con sus compañeros.

- Introducir métodos y técnicas apropiados a los alumnos con N.E.E.

- Utilizar técnicas, procedimientos e instrumentos de evaluación adecuados.

- Incorporar en las actividades de aprendizaje las ayudas y apoyos necesarios.

- Eliminar actividades en las que los alumnos con N.E.E. no se beneficien.

- Introducir actividades, individuales y grupales, para conseguir objetivos comunes al grupo de referencia.

\section{Adaptación de los contenidos}

No todos los contenidos han de ser iguales, ni el modo en el que se reflejen en las programaciones de aula ha de ser idéntico para todos los alumnos. De este modo, y teniendo en cuenta las capacidades y características personales de cada alumno, se procederá a:

- La secuenciación de contenidos: con el conocimiento a priori de las habilidades y conocimientos de cada alumno, se podrá organizar el orden de presentación y desarrollo de los contenidos.

- La modificación de la temporalización: respetando el ritmo de aprendizaje de cada alumno, se le concederá el tiempo suficiente para la realización de sus actividades y tareas.

- La priorización de contenidos: siempre y cuando se respete el equilibrio entre los conceptos, procedimientos y actitudes, se podrá determinar la primacía de unos contenidos sobre otros.

- La inclusión de nuevos contenidos: en algunas ocasiones será conveniente introducir nuevos contenidos para que un alumno, varios o todos puedan acceder al currículo.

- La exención de contenidos: en ocasiones se puede suprimir un área o varias del currículo o adaptar de modo muy significativo el contenido de un/as área/as, siempre y cuando estas modificaciones no afecten el que el alumno pueda lograr los objetivos generales de etapa.

\section{Adaptación en la evaluación}

La evaluación, al igual que los otros elementos curriculares, también es susceptible de ser modificada. Se ha de pensar que si se han modificado alguno/s elemento/s básicos curriculares, es necesario obligatoriamente modificar la evaluación. Ésta puede ser:

- Procesual: no sólo nos ocupamos de los resultados obtenidos, sino del proceso de enseñanza-aprendizaje que el alumno ha seguido, deteniéndonos en averiguar las dificultades que el alumno ha presentado.

- Formativa: se trata de que la evaluación no sea sancionadora, sino todo lo contrario; ayudamos al alumno a superar las dificultades que ha tenido.

- Criterial: considera al alumno como su propio referente, teniendo en cuenta su situación de partida, la evolución sufrida y la situación final a la que ha llegado tras el proceso de enseñanza-aprendizaje.

Algunas sugerencias para adaptar la evaluación serían:

- Establecer criterios de evaluación en cada una de las áreas curriculares que permitan evaluar a todos los alumnos, incluidos los que presenten necesidades educativas especiales. 
- Establecer criterios objetivos para la promoción de los alumnos.

- Decidir y consensuar las técnicas, procedimientos y estrategias generales de evaluación en el centro, teniendo en cuenta la presencia de alumnos con necesidades educativas especiales.

- Tener en cuenta a los alumnos con necesidades educativas especiales a la hora de elaborar instrumentos de evaluación.

- Elaborar modelos de informes que superen la tradicional concepción de calificación y categorización de los alumnos, y que permitan informar sobre los objetivos que se han planteado para el alumno y el grado de consecución de los mismos.

\subsection{Procedimiento, registro y seguimiento}

Cuando a un alumno se le haya realizado una adaptación curricular no significativa y haya sido insuficiente para su aprendizaje, el profesor tutor y profesor de apoyo, a partir de una evaluación psicopedagógica, considerarán la conveniencia de realizar una adaptación significativa.

Recopilada toda la información, se celebrará una reunión en el centro en la que jefe de estudios, profesor tutor y de apoyo, profesores implicados y profesionales, decidirán la pertinencia o no de la adaptación y de su alcance.

En caso afirmativo, se procederá a elaborar una adaptación curricular individualizada en la que intervendrán el profesor tutor, de apoyo, los profesores que vayan a intervenir con el alumno y el EOEP.

El registro de las adaptaciones curriculares realizadas tendrá lugar en el Documento Individual de Adaptación Curricular (DIAC) que se estructurará de la siguiente manera: datos de identificación del alumno, datos de identificación del documento, datos relevantes para la toma de decisiones (el nivel de competencia curricular, el estilo de aprendizaje y el contexto escolar), necesidades educativas especiales que presenta, propuesta de adaptaciones que necesita, como de acceso y de currículo, modalidad de apoyo, pautas de colaboración con la familia, criterios de promoción y seguimiento.

Una vez elaborado el DIAC (Documento Individual de Adaptación Curricular), el director del centro lo enviará al Servicio de Inspección Técnica Educativo (SITE), el cual emitirá un informe, ya sea favorable o desfavorable.

EI DIAC, el informe emitido por la Inspección y los resultados de la evaluación psicopedagógica habrán de incluirse en el expediente académico del alumno.

De forma paralela, la familia irá recibiendo toda la documentación del alumno; en caso que no estén de acuerdo con la adaptación o con algún aspecto, podrán manifestar su reclamación al director del centro y al Servicio de Inspección.

Este tipo de adaptaciones tendrán una duración mínima de un ciclo educativo, siendo necesario una nueva aprobación cuando se produzca una modificación de ciclo. Una vez que el alumno supere el ciclo, promocionará de acuerdo a la normativa vigente, y se analizará la conveniencia de continuar con la adaptación o de suprimirla.

\section{CONCLUSIÓN}

Se ha visto cómo el proceso de atención a la diversidad escala entre los diferentes niveles de concreción curricular, que se plantean en la actualidad en nuestro Sistema Educativo. En este proceso, es clave el concepto de Necesidades Educativas Especiales que pone el énfasis educativo en obtener el máximo desarrollo de las potencialidades del alumno a partir de una adaptación adecuada del contexto educativo.

En esta línea, los centros educativos asumen y llevan a cabo los principios de integración, normalización y no discriminación referidos a todo el alumnado y específicamente al que presenta n.e.e. La ley educativa actual, LOMCE, entendida como un modelo de escuela flexible, capaz de dar cabida a las necesidades de distintos estudiantes de una forma natural, una escuela donde quepa y aporte todo el mundo, sin importar demasiado sus diferencias.

Un aspecto a destacar, es la organización de los recursos, tanto materiales como personales, sobre todo en la respuesta educativa, que ha de preverse ya desde el propio Proyecto Educativo de Centro y en particular desde sus Concreciones Curriculares. A su vez, una buena atención a la diversidad, requiere una excelente coordinación y consenso en cuanto a las vías de colaboración entre los distintos profesionales, y a la elección de los recursos más adecuados para atender a estos 
alumnos. En la elección de los materiales, hay que tener en cuenta que son instrumentos para facilitar el proceso de enseñanza-aprendizaje y que nunca pueden suponer una dificultad añadida.

\section{Bibliografía}

- Alcudia y otros (2000). La atención a la diversidad. Madrid: Grao.

- Bautista, R. (2002). Necesidades educativas especiales. Archidona (Málaga): Aljibe.

- De Carlos García, Á., Arregi Martínez, A., Rubio Carcedo, T. (1996). Las necesidades educativas especiales en la educación primaria. Vitoria-Gasteiz : Servicio Central de Publicaciones del Gobierno Vasco. 